\title{
Overcoming dream-reality confusion using a complex numeric data generator
}

\author{
Alexey I. Chizhik \\ Georg-August-University Göttingen, Third Institute of Physics, Friedrich-Hund-Platz 1, 37077 \\ Göttingen, Germany
}

Phone: +49-551-39-27723

Email: alexey.chizhik@phys.uni-goettingen.de

The author declares no conflict of interests.

Author contribution: all the work was done by AIC

\section{Abstract}

This paper presents a method that allows a patient to distinguish whether a current experience is dreamt or real. The method is based on mathematical analysis of complex data that are generated by an external object (for instance, a computer or another person). High complexity of the data and possibility to quantitatively analyze it allow one to unambiguously disentangle dream-reality confusion. The author hopes that the method will help patients that experience lucid dreaming and suffer from dream-reality confusion.

Keywords: lucid dreams, dream-reality confusion, recognition, consciousness, cognitive disturbances. 
High complexity of dreams interpretation and their private character impedes their objective analysis and can lead to confusion of dreamt and real events. In some cases, this entanglement can take the form of dream-reality confusion (DRC) which is a difficulty or inability to determine whether an event happened or is currently happening during the waking state or if it was a part of a dream(Skrzypińska and Szmigielska 2015; Skrzypińska et al. 2018). This phenomenon is familiar to those who experience so called lucid dreams (Voss et al. 2014; Christoff et al. 2018; Voss et al. 2009), where one realizes that he or she is dreaming. Confusion of dreams and reality can have severe consequences for the interpretation of future real experiences as well as for interpersonal interactions (Hempel et al. 2003; Skrzypińska and Szmigielska 2015). Despite a significant number of studies on DRC, there is still no consensus on reliable mechanism for classification of real and dreamt events (Kühle 2015; Horton 2011; Kemp et al. 2003).

While sleeping, the enormous potential of the human brain allows us to create an imaginary world and to perceive it simultaneously and instantaneously. A possible way to distinguish dreams from reality is to unambiguously separate the processes of creation and perception of environment. The main obstacle for this approach is that our brain can generate nearly the whole spectrum of senses that we typically perceive in the real world (Fig. 1, upper panel). Therefore, the method for unambiguous separation of dream from reality should be based on generation of information that is (i) very unlikely to be spontaneously created by the brain during sleep and (ii) that can be quantitatively analyzed. In contrast to qualitative description of dreamt experiences, quantitative analysis of a certain information can be the key to unambiguous attribution of a dreamt and real experience. Both requirements can be satisfied by generating highly complex numeric data by an external object (another person or a device). Analysis of complex numeric data does not require any sophisticated equipment and its result cannot be instantly generated by the human brain, but rather requires significant effort.

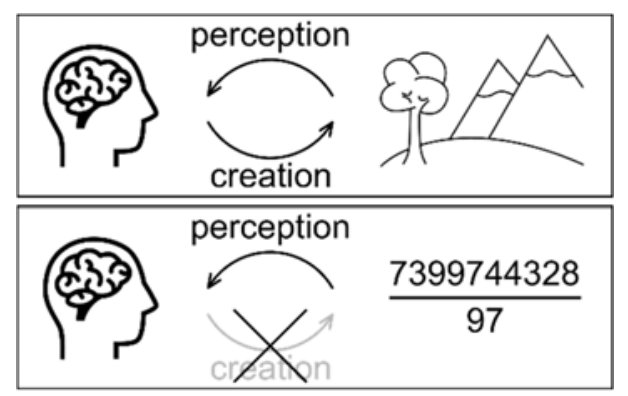

Fig. 1. Creation and perception of qualitative information (upper panel) versus perception of complex numeric data (lower panel) that can be generated only by an external object. 
Here, a mechanism to overcome DRC that is based on the human ability to analyze numeric data is proposed. The method is based on mathematical verification of complex data by the person (further attributed as "patient") that wants to discriminate whether what he is currently experiencing is dreamt or real. The patient obtains a number (or numeric data in a more general case) from another person or a device (for instance, a computer). The number that the patient obtains should possess a certain mathematical property, which should fulfil the following requirements:

1. Information about the property should be provided to the patient simultaneously with the information about the number.

2. It should be very unlikely for a random number to possess this property.

3. The property should be mathematically verifiable.

4. Verification of the property should require significant effort from the patient.

To unravel DRC, the patient should try to verify that the number indeed satisfies the property given. It is crucial that verification should be done directly by the person that wants to unravel DRC, but not using any auxiliary device (e.g. a computer or electronic calculator). The data that is generated to satisfy all the above parameters guarantees that it was produced not by the brain of the patient, but by an external object (a person or device) in the real world (Fig. 1, lower panel).

Let us consider a simple example of how the method works. A good example that does not require any specialized knowledge from the patient except the school math is determination whether a given integer is divisible by a certain divisor. Let us imagine that the patient simultaneously obtains a number 7399744328 and a statement that this number can be divided by 97 with no remainder. The probability for a random integer number to be divisible by 97 with no remainder is approximately $1 \%$, which is relatively low. Therefore, we can virtually exclude that one can randomly generate a number with this mathematical property. The divisibility of 7399744328 by 97 can be mathematically verified by implementation the division, for instance, using long division. Finally, division of 7399744328 by 97 are impossible for mental math for the vast majority of people. This assures that the person needs significant effort to verify the divisibility. Thus, the number and the property of the number that the person obtained satisfy all the above requirements. As a result, it is very unlikely that the brain of the person can generate the data of such level of complexity. Hence, the person makes sure that it was generated by an external object.

Besides analysis of mathematical data, one can consider experimental verification of e.g. physical or chemical parameters of an object. However, this may either require complicated equipment or lead to significant errors of measurements. This can hinder precise verification of the 
data and thus prevent reliable unravel of DRC. The author hopes that the method proposed in this work will help patients that have difficulties in distinguishing dreams and reality to unravel this confusion.

\section{Acknowledgements}

The author thanks Christopher Noland for inspiring him for this work.

\section{References}

Christoff, K., K. C. R. Fox, J. M. Windt, and U. Voss. 2018. Spontaneous Thought, Insight, and Control in Lucid Dreams: Oxford University Press.

Hempel, A. G., A. R. Felthous, and J. R. Meloy. 2003. Psychotic dream-related aggression: A critical review and proposal. Aggression and Violent Behavior 8 (6):599-620.

Horton, C. L. 2011. Recall and recognition of dreams and waking events: A diary paradigm. 2011:9.

Kemp, S., C. D. B. Burt, and M. Sheen. 2003. Remembering dreamt and actual experiences. Applied Cognitive Psychology 17 (5):577-591.

Kühle, L. 2015. Insight: What Is It, Exactly? In Open MIND, edited by T. K. Metzinger and J. M. Windt. Frankfurt am Main: MIND Group.

Skrzypińska, D., M. Hołda, B. Szmigielska, and M. Słodka. 2018. The phenomenology of dream-reality confusion: A quantitative study. Dreaming 28 (3):245-260.

Skrzypińska, D., and B. Szmigielska. 2015. Dream-reality confusion in borderline personality disorder: a theoretical analysis. Frontiers in Psychology 6 (1393).

Voss, U., R. Holzmann, A. Hobson, W. Paulus, J. Koppehele-Gossel, A. Klimke, and M. A. Nitsche. 2014. Induction of self awareness in dreams through frontal low current stimulation of gamma activity. Nature Neuroscience 17:810.

Voss, U., R. Holzmann, I. Tuin, and A. J. Hobson. 2009. Lucid Dreaming: a State of Consciousness with Features of Both Waking and Non-Lucid Dreaming. Sleep 32 (9):1191-1200. 